\title{
Hematoma crónico de páncreas. Diagnóstico diferencial con tumor maligno. Informe de un caso
}

\author{
Dra. Belinda Rafael-Alemán, ${ }^{*}$ Dr. Sergio Terriquez-Rodríguez, ${ }^{* *}$ Dra. Sandra F. Páez-Aguirre, ${ }^{* * *}$ Dr. Pedro \\ Salvador Jiménez-Urueta**
}

\section{RESUMEN}

Introducción. Los hematomas crónicos del páncreas en niños son raros y de difícil diagnóstico. Los diagnósticos diferenciales más relevantes son: seudoquiste de páncreas cuando existe el antecedente de traumatismo, y tumor seudopapilar de páncreas cuando dicho antecedente no existe. El estudio de imagen ideal es la tomografía de abdomen. En el caso que presentamos el diagnóstico definitivo fue quirúrgico mediante biopsia transoperatoria.

Informe del caso. Se presenta el caso de una adolescente de 14 años de edad con antecedente de traumatismo abdominal cerrado cuatro meses antes del inicio de su padecimiento actual, caracterizado por: dolor en abdomen superior, náuseas, vómito y pérdida de peso de $8 \mathrm{Kg}$. La tomografía reveló lesión quística en cabeza de páncreas. Con sospecha de seudoquiste de páncreas vs tumor seudopapilar se sometió a laparotomía exploradora. Durante la cirugía se descubrió hematoma capsulado de la cabeza de páncreas realizándose vaciamiento y ventana a peritoneo, así como toma de biopsia. La evolución postoperatoria fue adecuada.

Discusión. El hematoma de páncreas es raro y de difícil diagnóstico. Los diagnósticos diferenciales más importantes son seudoquiste y tumor seudopapilar de páncreas.

Palabras clave: Hematoma pancreático, tumor seudopapilar del páncreas, biopsia transoperatoria, traumatismo.

\section{ABSTRACT}

Introduction: Chronic hematomas of the pancreas are rare and difficult to diagnose in children. The most relevant differential diagnosis include: pancreatic pseudocyst (when a history of trauma is referred) and pseudopapillary tumor of the pancreas (when a history of trauma is denied). The gold standard study imaging consists of an abdominal CT scan. In the case we present, the diagnosis was not made by $\mathrm{CT}$, and could only be obtained after surgical biopsy.

Case report. A case of a female adolescent with history of closed abdominal trauma 4 months before the onset of symptoms such as upper abdominal pain, nausea, vomiting, and weight loss of $8 \mathrm{~kg}$. The CT image only revealed a cyst of the head of the pancreas. The differential diagnosis included: pancreatic pseudocyst vs. a malignant tumor. Surgery was indicated. During surgery, a pancreatic hematoma of the head of the pancreas was diagnosed, drained and marsupialized. The postoperative outcome was uneventful.

Discussion. Hematomas of the pancreas are rare and difficult to diagnose. The most relevant differential diagnosis include: pancreatic pseudocyst and pseudopapillary tumor of the pancreas.

Key words: Pancreatic hematoma, solid-cystic pseudopapillary tumor of pancreas, surgical biopsy, trauma.

* Servicio de Pediatría

** Médico Adscrito al Servicio de Cirugía Pediátrica

*** Médico adscrito al Servicio de Oncología Pediátrica C.M.N. "20 de Noviembre" ISSSTE

Correspondencia: Dra. Belinda Rafael-Alemán. Centro Médico Nacional 20 de Noviembre ISSSTE. Felix Cuevas 540, colonia Del Valle, México, 03100, DF.

Recibido: noviembre, 2009. Aceptado: febrero, 2010.

Este artículo debe citarse como: Rafael-Alemán B, Terriquez-Rodríguez S, Páez-Aguirre SF y col. Hematoma crónico de páncreas. Diagnóstico diferencial con tumor maligno. Informe de un caso. Acta Pediatr Mex 2010;31(2):64-66.

\section{www.nietoeditores.com.mx}

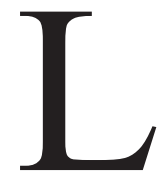

as lesiones pancreáticas ocurren en menos del 5 $\%$ de los traumatismos mayores abdominales ${ }^{1}$. Dos tercios están asociadas con trauma abdominal penetrante y un tercio con trauma abdominal contuso cerrado ${ }^{2}$. La situación anatómica profunda, retroperitoneal y central del páncreas lo protegen habitualmente de las lesiones postraumáticas ${ }^{3}$. Las lesiones se presentan por compresión del páncreas contra la columna vertebral, siendo difíciles de detectar sin estudios de imagen ${ }^{4}$, pueden variar de contusiones menores y hematomas, a laceración mayor o fracturas asociadas a lesión de los ductos pancreáticos. 
La morbilidad y mortalidad se incrementan con daño a otros órganos y estructuras vasculares, el retraso en el diagnóstico y la intensidad del traumatismo ${ }^{4}$. La presentación clínica de las lesiones pancreáticas puede variar desde la estabilidad hemodinámica hasta una situación de choque. Debido a su localización retroperitoneal, la detección precoz de la lesión pancreática basada únicamente en los datos clínicos puede ser complicada, ya que la irritación peritoneal suele ocurrir tardíamente y hacerse evidente sólo cuando la sangre, la bilis o las enzimas pancreáticas se extravasan a la cavidad abdominal. Cualquier paciente que presente molestias en el cuadrante superior derecho y epigastrio debe ser evaluado cuidadosamente bajo la sospecha de una lesión pancreática subyacente ${ }^{5,6}$. En esta paciente los diagnósticos considerados fueron tumor sólido seudopapilar, seudoquiste y hematoma peripancreático. La dificultad del diagnóstico clínico y de imagen hace relevante la experiencia obtenida en este caso. No obstante que su diagnóstico preciso es difícil, habitualmente la solución es sencilla. Realizamos un análisis de las imágenes y el comportamiento clínico del tumor seudopapilar quístico y las comparamos con el caso de nuestra paciente.

\section{PRESENTACIÓN DEL CASO}

Niña de 14 años de edad. Antecedentes de abuela materna con tumor quístico gástrico. Sufre traumatismo directo en epigastrio, y después de un periodo asintomático de cuatro meses inicia su padecimiento en forma súbita con dolor abdominal en epigastrio y mesogastrio, de moderada intensidad, irradiado en cinturón, asociado a vómitos postprandiales mediatos de contenido gástrico, y sensación de plenitud postprandial temprana, de predomino matutino; pérdida de peso de $8 \mathrm{~kg}$. Exploración física. Obesidad exógena, a nivel abdominal panículo adiposo abundante, dolor a la palpación media y profunda en epigastrio y mesogastrio; se palpa masa en hipocondrio derecho, por debajo del borde costal, peristalsis presente, sin datos de irritación peritoneal. Resto de la exploración normal. Biometría hemática. Leucocitosis de 14,590, neutrófilos totales $67.1 \%$; química sanguínea, electrólitos séricos, tiempos de coagulación, pruebas de función hepática, amilasa, lipasa e insulina, perfil de lípidos, dentro de límites normales para la edad.

Ultrasonido abdominal: imagen redondeada de contenido anecoico, a nivel del proceso uncinado del páncreas, de contorno discretamente irregular, delimitada por una pared, con múltiples septos en su interior; dimensiones de $5.8 \times 5.8$ x $5.6 \mathrm{~cm}$. Tomografía computada simple y contrastada de abdomen: lesión hipodensa, redondeada, de bordes bien definidos, de 5 x $5 \mathrm{~cm}$, localizada en la cabeza del páncreas (Figura 1).
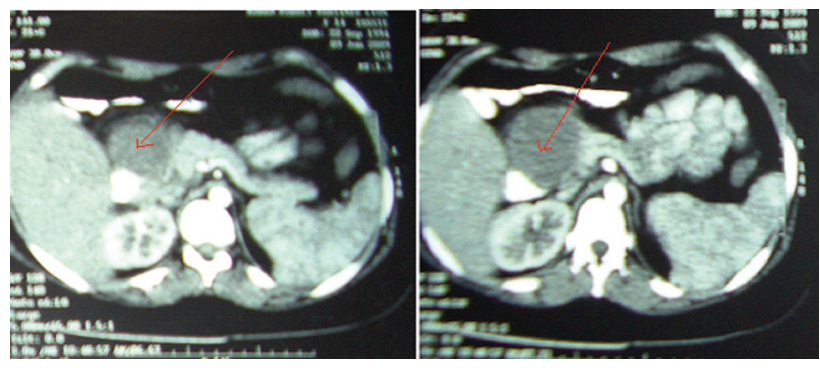

Figura 1. Imagen quística hipodensa de la cabeza del páncreas, con cápsula bien definida.

Con las posibilidades diagnósticas de seudoquiste o quiste simple de páncreas vs tumor seudopapilar de páncreas, se sometió a laparotomía exploradora. Durante la cirugía se encontró hematoma localizado en la cabeza del páncreas, de aproximadamente $6 \mathrm{~cm}$ de diámetro, el cual se drenó sin complicaciones; se tomó biopsia de la pared (Figura 2). La evolución postoperatoria fue satisfactoria. El estudio histopatológico, transoperatorio y definitivo mostró: fibrosis e inflamación crónica, sin datos de malignidad.

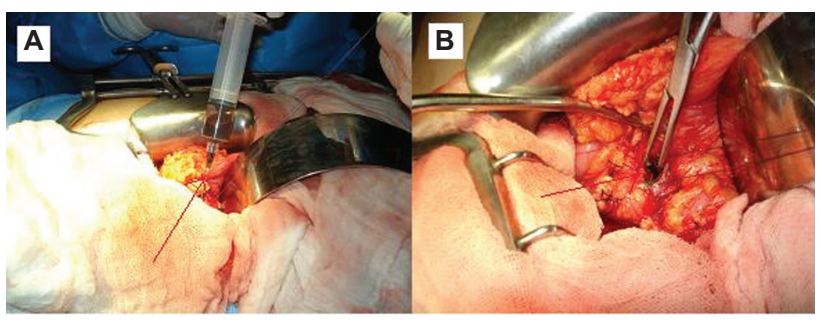

Figura 2. A. Punción de la masa obteniéndose sangre. B. Se realizó ventana y drenaje total con toma de biopsia de pared.

\section{DISCUSIÓN}

Las lesiones traumáticas del páncreas son raras en niños y de difícil diagnóstico. Bosbomm en un estudio retrospectivo en niños con trauma abdominal cerrado encontró 34 con daño pancreático, sólo un hematoma de cabeza de páncreas 7. El antecedente lejano de traumatismo, el género, edad y cuadro clínico, así como las características tomográficas, en este caso, desviaron la atención hacia la posibilidad de 
una neoplasia benigna. En la paciente se sospechó tumor seudopapilar de páncreas o tumor Gruder-Frantz, raro, de bajo potencial maligno que ocupa el $6 \%$ de los tumores exocrinos del páncreas, y $5 \%$ de las neoplasias quísticas del páncreas, ocurre casi exclusivamente en niñas adolescentes y debe diferenciarse de otras patologías pancreáticas como seudoquistes ${ }^{8}$. Se presentan como una tumoración abdominal dolorosa, su apariencia en estudios de imagen es característica, como una masa bien encapsulada, con áreas de calcificación y degeneración hemorrágica así como cambios quísticos degenerativos ${ }^{9}$. El diagnóstico depende de la confirmación histológica.

La sintomatología frecuentemente se relaciona al tamaño del tumor. La excisión quirúrgica es el tratamiento curativo en muchos casos, con un pronóstico bueno después de la resección ${ }^{10}$. Lee encontró una frecuencia del $66.7 \%$ de tumor seudopapilar localizado en la cabeza del páncreas ${ }^{11}$. El término tumor sólido-seudopapilar del páncreas fue recientemente introducido. El diagnóstico diferencial debe hacerse con cualquier enfermedad pancreática sólida o quística, como tumor mucinoso quístico, adenoma microquístico, tumor de células de los islotes, cistoadenocarcinoma, carcinoma de células acinares, seudoquiste inflamatorio, tumor secretor de moco, pancreatoblastoma y tumores vasculares; todos raros en el paciente pediátrico ${ }^{9}$. Las lesiones pancreáticas aisladas resultantes de traumatismo no penetrante son raras, se presentan del 2 al $12 \%$. La tomografía es actualmente el estudio de elección en la evaluación de la lesión pancreática. El retraso en el reconocimiento de los pacientes que necesitan una intervención inmediata es una causa importante de incremento en la morbilidad debida a complicaciones pancreáticas específicas ${ }^{6}$. Los datos clínicos y de gabinete, así como la investigación de los tumores de páncreas hicieron sospechar de inicio un tumor seudopapilar de páncreas vs seudoquiste. La paciente, después del traumatismo, estuvo cuatro meses asintomática, por lo que el hematoma peripancreático fue una posibilidad diagnóstica remota. La resolución quirúrgica consistió en drenaje del hematoma, toma de biopsia y realización de una ventana para drenaje externo. La evolución postoperatoria fue satisfactoria. El pronóstico en el caso de hematomas de páncreas depende del comportamiento y el seguimiento; estos pacientes pueden desarrollar seudoquiste de páncreas. Hasta el momento nuestra paciente no ha tenido complicaciones y se encuentra asintomática. Desafortunadamente la literatura nacional como la internacional no es muy abundante en el tema, la casuística mayor donde se informa de los hematomas de páncreas es la revisada por Bosbomm, donde además demuestra la rareza del hematoma pancreático con un caso de 35 lesiones de páncreas ${ }^{7}$. En las revisiones de la literatura mexicana no encontramos una fuente que hable específicamente de hematoma pancreático ya que es rara esta forma de presentación. La cirugía y la indicación de la misma están dentro de los parámetros éticos ya que en realidad nunca se sospechó un hematoma; el diagnóstico más importante para el grupo fue el de tumor seudopapilar de páncreas. En retrospectiva es factible realizar un diagnóstico laparoscópico con toma de biopsia de la lesión; sin embargo, el clínico debe ser cuidadoso para definir cuál es la conducta más apropiada y útil para el paciente.

\section{REFERENCIAS BIBLIOGRAFICAS}

1. Craig MH, Talton DS, Hauser CJ, Poole GV. Pancreatic injuries from blunt trauma. Am Surg. 1995;61(2):125-8.

2. Yeo CJ, Cameron JL. Exocrine pancreas. In: Townsend CM, Beauchamp RD, Evers BM, Mattox KL, eds. Sabiston Textbook of Surgery, 16th ed. Singapore: Harcourt Asia; 2001. p. 1112-43.

3. Khanna LT, Col V, Gualti LT, Col YS, Col MN, Sharama R. A rare case of pancreatic injury: case report with an imaging perspective. Asian Oceanian J Radiol. 2004;9(1):64-7.

4. Mahale A, Gupta A, Paudel K. A rare case of pancreatic injury: A case report. Indian J Radiol Imaging. 2006;16(2):437-9.

5. Petrone P, Asensio JA, Pardo M, Kimbrell B,Kuncir E. Traumatismos de páncreas: diagnóstico y tratamiento. Rev Coloma Cir. 2008;23(1):42-52.

6. Brestas PS, Karakyklas D, Gardelis J, Tsouroulas M, Drossos C. Sequential CT evaluation of isolated non-penetrating pancreatic trauma. JOP. J Pancreas. 2006;7(1):51-5.

7. Bosboom D, Braam AW, Blickman JG, Wijnen RM. The role of imaging studies in pancreatic injury due to blunt abdominal trauma in children. Eur J Radiol. 2006;59(1):3-7.

8. Chung EM, Travis MD, Conran RM. Pancreatic tumors in children: radiologic-pathologic correlation. Radiographics. 2006;26(4):1211-38.

9. Casanova M, Collini P, Ferrari A, Cecchetto G, Dall'Igna P, Mazzaferro V. Solid pseudopapillary tumor of the pancreas (Frantz tumor) in children. Med Pediatr Oncol. 2003;41(1):746.

10. Vargas B, Dominguez E, Chinchon D. Four cases of solid pseudopapillary tumors of pancreas: imaging findings and pathological correlations. Eur J Radiol. 2006;58(1):132-9.

11. Lee SE, Jang JY, Hwang DW, Park KW, Kim SW. Clinical features and outcome of solid pseudopapillary neoplasm: differences between adults and children. Arch Surg. 2008;143(12):1218-21. 